\title{
Dépasser le gap entre production de savoirs scientifiques et utilisation par les praticiens
}

\author{
Stéphane Fukazawa-Couckuyt
}

Stéphane Fukazawa-Couckuyt.

Résumé. La formation des entraîneurs peut impacter les apports qu'ils font aux sportifs. Cet article vise caractériser le décalage (gap) entre la production de savoirs scientifiques et l'appropriation/utilisation par les praticiens, ainsi qu'à identifier des leviers opérationnels pour le chercheur et l'entraîneur visant à réduire ce gap. Le cadre de Finch est utilisé, structurant le propos autour de trois métacatégories sources du gap : la recherche peut être non pertinente, ne pas atteindre les entraîneurs, ou encore les atteindre mais avec un impact faible. Après une présentation synthétique des facteurs et leviers dans chaque catégorie, la possibilité de développer des rôles de "passeurs » entre monde de la recherche et monde de l'intervention sportive est abordée, ainsi que d'agir sur la réduction du gap en travaillant davantage sur l'expérience et la science comme sources complémentaires d'apprentissage pour l'entraîneur.

Mots-clés. Gap, Science, Praticien, Translation, Savoir

\section{Introduction}

S'interroger sur les apports des entraîneurs aux sportifs amène à s'intéresser à leur formation tant formelle qu'informelle (Brougère \& Bézille, 2007), médiée que non médiée (Werthner \& Trudel, 2006). Les savoirs issus de la science peuvent contribuer à cette formation, bien que des études pointent la faible contribution de ces savoirs à l'agir des entraîneurs (p. ex. Reade, Rodgers \& Hall , 2008) et l'existence d’un fossé (« gap ») depuis au moins 40 ans entre production de savoirs scientifiques et mise en œuvre par les entraîneurs sportifs (Gould, 2016; Haugen, 2019; Reade, Rodgers \& Spriggs, 2008).

Le sujet est d'actualité et ne peut être ignoré pour une meilleure translation/implémentation (Eisenmann, 2017; Finch, 2011) des savoirs déjà produits et des savoirs à venir. En effet, d'une part, récemment Gould (2016), consultant pour les fédérations olympiques américaines et chercheur, expliquait que la plupart des questionnements des entraîneurs ne nécessite pas fondamentalement de nouveaux savoirs scientifiques mais que l'enjeu majeur est la circulation/ appropriation des savoirs existants. D’autre part, plusieurs sociétés savantes, dont l'ARIS, témoignent de la vitalité de la recherche sur le sport, tandis que l'obtention de l'organisation des Jeux olympiques de 2024 par la France est accompagnée d'un budget supplémentaire alloué sous forme d'appel à projets de recherche et donc une augmentation de la production de savoirs scientifiques, qui doivent servir aux entraîneurs et donc dépasser le gap.

Au-delà de son écho contemporain, le gap questionne la place du savoir et notamment du savoir issu de la recherche, dans l'agir de l'entraîneur. Dans la société du savoir qui caractérise le nouveau cycle post-industriel (David \& Foray, 2002), le savoir est le principal capital. Les entraîneurs étant intéressés par les savoirs scientifiques (Collinet, 2005; Reade, Rodgers \& Spriggs, 2008), il y a donc des enjeux à ne pas ignorer ce gap, à le caractériser et à déterminer les leviers disponibles par le chercheur et l'entraîneur en vue de le réduire. Quelles sont les raisons du faible apport des savoirs scientifiques aux entraîneurs sportifs ? Comment agir sur cette situation?

Cette distanciation entre savoirs scientifiques et usage par les praticiens n'est pas spécifique au milieu de l'entraînement. Elle se retrouve en formation des adultes (Mezirow, 2001), en travail infirmier (Bishop, 2008), dans les pratiques de gestion (Schmitt, 2007), par exemple. Si dans de nombreux domaines les rapports entre les chercheurs et les praticiens sont souvent ambigus et contradictoires (Lacourse \& Thibault, 1996; Vinatier \& Rinaudo, 2015), cette explication reste partielle. Nous mobilisons le cadre de Finch (2011) qui distingue trois métacatégories explicatives de ce gap. 
Dépasser le gap entre production de savoirs scientifiques et utilisation par les pratic...

La recherche peut être hors de propos par rapport au champ d'activité, à la réalité de l'entraînement et du métier (research relevance failure). Elle peut ne pas atteindre son public (dissemination failure). Enfin, il est possible que la recherche atteigne le public mais avec un faible impact (translation/adoption failure). Dans la suite du texte, nous proposons un tableau pour chacune de ces catégories. Conçus à partir de la littérature, ces tableaux visent à donner au chercheur et à l'entraîneur une vision synthétique et opérationnelle des facteurs du gap et des leviers mobilisables. Dans ces tableaux, les leviers relevant plutôt du chercheur, de l'entraîneur ou d'une autre personne ou institution sont identifiés respectivement par « [C]» «[E]» et « [?]».

\section{Premier objectif : produire des savoirs pertinents pour les entraîneurs sportifs}

Le tableau 1 décrit les facteurs du gap et les leviers relatifs à la pertinence des savoirs scientifiques pour un public d'entraîneurs.

Tableau 1 : Les facteurs "research relevance failure" et leurs leviers

\begin{tabular}{|c|c|c|}
\hline $\begin{array}{l}\text { Facteurs de cette } \\
\text { catégorie }\end{array}$ & Leviers & Sources \\
\hline $\begin{array}{l}\text { Certaines postures } \\
\text { scientifiques : } \\
\text { Vision analytique, } \\
\text { réductionniste, de } \\
\text { rationalité technique, } \\
\text { réification... } \\
\text { Ne sont pas cohérentes } \\
\text { avec la réalité de } \\
\text { l'entraînement sportif: } \\
\text { Intervention systémique, } \\
\text { singulière, dans } \\
\text { l'adaptation, la gestion } \\
\text { des imprévus, des } \\
\text { dilemmes... }\end{array}$ & $\begin{array}{l}\text { [C] Adopter une approche } \\
\text { systémique } \\
\text { [E/C] S'acculturer } \\
\text { réciproquement, dès la } \\
\text { formation initiale } \\
\text { [E/C] Positionner l'interaction } \\
\text { chercheurs-entraîneurs sur un } \\
\text { pied d'égalité, de sachant à } \\
\text { sachant, en co-construction } \\
\text { [E/C] Réfléchir davantage sur } \\
\text { les savoirs à produire et les } \\
\text { savoirs produits / prise en } \\
\text { compte des besoins }\end{array}$ & $\begin{array}{l}\text { Bernardeau-Moreau \& Collinet, 2009; Buchheit, 2017; Couckuyt, } \\
\text { Robin \& Sarremejane, 2016; Eisenmann, 2017; Finch, 2011, p. 201; } \\
\text { Fleurance, 2006; Fleurance \& Pérez, 2008; Lacourse \& Thibault, 1996; } \\
\text { Mouchet, 2018; Pérez, 2009; Rolland \& Cizeron, } 2011\end{array}$ \\
\hline
\end{tabular}

\section{Deuxième objectif : favoriser la circulation des savoirs scientifiques jusqu'au public- cible}

Le tableau 2 décrit les facteurs du gap et les leviers relatifs à la diffusion des savoirs scientifiques auprès des entraîneurs. 
Actes de la 11ème Biennale de l'ARIS : Former des citoyens physiquement éduqués. Axe 3 - Au-delà de la performance, $10.25518 /$ sepaps 20.461

Tableau 2 : les facteurs "dissemination failure" et leurs leviers

\begin{tabular}{|l|l|l|}
\hline Facteurs de cette catégorie & Leviers & Sources \\
\hline Manque d'interaction & $\begin{array}{l}\text { [E/C] Avoir la possibilité d'accéder } \\
\text { aux uns et aux autres, dans un } \\
\text { environnement propice }\end{array}$ & $\begin{array}{l}\text { Cushion, Armour \& Jones, 2003; Jones et al., 2019; } \\
\text { Lacourse \& Thibault, 1996; Reade, Rodgers \& Hall, 2008; } \\
\text { Reade, Rodgers \& Spriggs, 2008 }\end{array}$ \\
\hline $\begin{array}{l}\text { Difficulté d'accès aux savoirs } \\
\text { issus de la science, relation en } \\
\text { sens quasiment unique }\end{array}$ & $\begin{array}{l}\text { [C] Dégager du temps pour la } \\
\text { circulation } \\
\text { [E] S'entraider, partager entre pairs } \\
\text { les articles et savoirs scientifiques } \\
\text { [?] Dégager un budget pour l'accès à } \\
\text { la science }\end{array}$ & $\begin{array}{l}\text { Cloes, lenzen \& Trudel, 2009; Eisenmann, 2017; Finch, } \\
\text { 2011; Gould, 2016; Schmitt, 2007 }\end{array}$ \\
\hline
\end{tabular}

Troisième objectif: outiller les entraîneurs sportifs, impacter les pratiques d'intervention

Le tableau 3 décrit les facteurs du gap et les leviers relatifs à l'appropriation des savoirs scientifiques par les entraîneurs. 
Dépasser le gap entre production de savoirs scientifiques et utilisation par les pratic...

Tableau 3 : les facteurs "translation/adoption failure" et leurs leviers

\begin{tabular}{|c|c|c|}
\hline Facteur GAP & Leviers & Sources \\
\hline $\begin{array}{l}\text { Manque de langage partagé / savoirs rédigés } \\
\text { pour des pairs, non adressés au public cible (les } \\
\text { entraîneurs) } \\
\text { Overdose d'informations pour le praticien, voire } \\
\text { contradiction entre elles. Où chercher, quoi } \\
\text { retenir? }\end{array}$ & $\begin{array}{l}\text { [C/ ?] Eduquer les futurs chercheurs à } \\
\text { écrire pour les entraîneurs, de } \\
\text { manière intelligible en présentant } \\
\text { différemment les mêmes savoirs, ce } \\
\text { qui suppose une traduction/mise en } \\
\text { scène } \\
\text { [E/C] Légitimer les savoirs } \\
\text { scientifiques par utilisation dans } \\
\text { divers contextes d'entraînement } \\
\text { [E] S'entraider, partager entre pairs } \\
\text { les articles et savoirs scientifiques } \\
\text { jugés pertinents } \\
\text { [E/C] Accompagner les entraineurs } \\
\text { grâce à des chercheurs qui orientent } \\
\text { et facilitent }\end{array}$ & $\begin{array}{l}\text { Avenier \& Schmitt, 2007; Bishop, 2008; } \\
\text { Buchheit, 2017; Cloes et al., 2009; } \\
\text { Cushion et al., 2003; Eisenmann, 2017; } \\
\text { Gould, 2016; Reade, Rodgers \& Hall, } \\
\text { 2008; Reade, Rodgers \& Spriggs, } 2008\end{array}$ \\
\hline $\begin{array}{l}\text { Manque de compétence pour comprendre avec } \\
\text { recul critique les écrits scientifiques }\end{array}$ & [E] Améliorer la formation & $\begin{array}{l}\text { Bishop, 2008; Eisenmann, 2017; Finch, } \\
2011\end{array}$ \\
\hline $\begin{array}{l}\text { Manque de marge de manœuvre des } \\
\text { entraîneurs (contextes contraints, complexité, } \\
\text { gestion d'imprévus et de dilemmes) }\end{array}$ & $\begin{array}{l}\text { [C] Prendre en compte les contextes et } \\
\text { contraintes, la réalité du métier }\end{array}$ & Fleurance, 2012; Gould, 2016 \\
\hline $\begin{array}{l}\text { Temporalités différentes qui perturbent } \\
\text { l'adoption des savoirs : praticiens dans des } \\
\text { méthodes rapides et informelles d'obtention de } \\
\text { savoirs, sous temps contraint VS chercheurs } \\
\text { dans temps long avec dilemme rigueur/ } \\
\text { pertinence }\end{array}$ & $\begin{array}{l}{[\mathrm{E} / \mathrm{C}] \text { Elaborer collectivement le }} \\
\text { dispositif de recherche ainsi que de la } \\
\text { temporalité des livrables } \\
\text { [E/C] Restituer les résultats en } \\
\text { articulant boucle courte/longue }\end{array}$ & $\begin{array}{l}\text { Biémar, Dejean \& Donnay, 2008; } \\
\text { Buchheit, 2017; Burlot et al., 2019; } \\
\text { Mouchet, 2018; Reade, Rodgers \& } \\
\text { Spriggs, 2008; Saury, } 2008\end{array}$ \\
\hline
\end{tabular}

\section{Conclusion}

Au-delà des constats, pour activer les leviers, un des éléments est la disponibilité des protagonistes. Du côté des chercheurs, cet article invite alors également à repenser la valorisation du «franchissement » du gap, par exemple dans le cadre de l'avancement de carrière, mais aussi de manière symbolique et culturelle. Possibilité complémentaire, une personne interface peut assurer cette translation en assurant « la traduction réciproque d'énoncés et de langages différents et la transaction entre des acteurs de mondes divers» (Lyet, 2011, p. 50). Elle doit connaître suffisamment les deux communautés et cultures pour favoriser les interactions, les translations réciproques et la compréhension partagée (Akkerman \& Bakker, 2011). Des initiatives commencent à poindre autour de la formation et de l'emploi de telles personnes. Par exemple, en France l'Institut National du Sport de l'Expertise et de la Performance (INSEP) porte depuis quelques années avec l'Université de Paris une formation de niveau Master visant justement à former 
Actes de la 11ème Biennale de l'ARIS : Former des citoyens physiquement éduqués. Axe 3 - Au-delà de la performance, 10.25518/sepaps20.461

ces personnes interfaces, les accompagnateurs scientifiques de la performance. Différents clubs professionnels ont également créé des cellules recherche en leur sein, avec des chercheurs au plus près des staffs et des collectifs d'entraînement pour optimiser la performance.

La littérature invite également à travailler sur le complexus expérience-savoir scientifique, sur les complémentarités (Trudel, Milestetd \& Culver, 2019). Cette thématique de recherche a déjà été abordée dans d'autres domaines, par exemple par Lièvre (2007) sur l'intégration de connaissances tacites et de savoirs scientifiques pour construire des savoirs pour l'action dans des situations extrêmes, notamment des expéditions polaires. En sciences du sport, Haugen (2019) souligne une approche norvégienne novatrice : les pratiques, méthodologies d'entraînement, savoirs et théories liés à l'expérience des meilleurs entraîneurs en endurance et en sprint sont testées scientifiquement et bien souvent validées. Ainsi, pour dépasser le gap une inversion de polarité est également une voie à investir.

Enfin, il existe également un gap entre formation des entraîneurs et activité sur le terrain, la formation étant relativement décriée comme ne préparant pas à la réalité (Bernardeau-Moreau \& Collinet, 2009; Cushion et al., 2003; Fleurance \& Pérez, 2008). Cette thématique pourrait constituer un objet de recherche ultérieure autour des facteurs et des leviers mobilisables par les ingénieurs de formation, les formateurs et les néo professionnels.

\section{Bibliographie}

Akkerman, S. F., \& Bakker, A. (2011). Boundary Crossing and Boundary Objects. Review of Educational Research, 81(2), 132-169. https://doi.org/10.3102/0034654311404435

Avenier, M.-J., \& Schmitt, C. (Eds.). (2007). La construction de savoirs pour l'action. Paris : L'Harmattan.

Bernardeau-Moreau, D., \& Collinet, C. (2009). Les éducateurs sportifs en France depuis 1945: Questions sur la professionnalisation. Rennes : Presses universitaires de Rennes.

Biémar, S., Dejean, K., \& Donnay, J. (2008). Co-construire des savoirs et se développer mutuellement entre chercheurs et praticiens. Recherche \& formation, (58), 71-84.

Bishop, D. (2008). An Applied Research Model for the Sport Sciences: Sports Medicine, 38(3), 253-263.

Brougère, G., \& Bézille, H. (2007). De l'usage de la notion d'informel dans le champ de l'éducation. Revue française de pédagogie, (158), 117-160.

Buchheit, M. (2017). Houston, We Still Have a Problem. International Journal of Sports Physiology and Performance, 12(8), 1111-1114. https://doi.org/10.1123/ijspp.2017-0422

Burlot, F., Delalandre, M., Joncheray, H., Demeslay, J., Julla-Marcy, M., Fukazawa-Couckuyt, S., \& Menon, P. (2019). Les conditions de travail des entraîneurs de haut niveau en France-Partie 1 Analyse qualitative (Rapport olympique d'enquête) (p. 94). Paris : Institut National du Sport, de l'Expertise et de la Performance.

Cloes, M., Lenzen, B. , \& Trudel, P. (2009). Analyse de la littérature francophone portant sur l'intervention de l'entraîneur sportif, publiée entre 1988 et 2007. Staps, (83), 7-23.

Collinet, C. (2005). Quels savoirs scientifiques les enseignants d'EPS et les entraîneurs jugent-ils utiles? Revue française de pédagogie, (150), 43-58.

Couckuyt, S., Robin, J.-F., \& Sarremejane, P. (2016). Des outils d'aide à la décision au sein du groupe d'entraînement en sport de haut niveau: Étude de cas. In Actes du colloque international « Décider en urgence » (p. 61-62). Créteil. 
Dépasser le gap entre production de savoirs scientifiques et utilisation par les pratic...

Cushion, C. J., Armour, K. M., \& Jones, R. L. (2003). Coach Education and Continuing Professional Development: Experience and Learning to Coach. Quest, 55(3), 215-230.

David, P. A., \& Foray, D. (2002). Une introduction à l'économie et à la société du savoir. Revue internationale des sciences sociales, 171(1), 13. https://doi.org/10.3917/riss.171.0013

Eisenmann, J. (2017). Translational Gap between Laboratory and Playing Field: New Era to Solve Old Problems in Sports Science. Translational Journal of the ACSM, 2(8), 37-43.

Finch, C. F. (2011). No longer lost in translation: The art and science of sports injury prevention implementation research. British Journal of Sports Medicine, 45(16), 1253-1257.

Fleurance, P. (2006). Rapport d'étude sur les référentiels professionnels et de formation des entraîneurs nationaux «Chef de projet performance» (p. 101). Ministère de la Jeunesse, des Sports et de la Vie Associative.

Fleurance, P. (2012). Au delà de la science normale? Pour de nouvelles relations entre les savoirs et l'action. Dossier MCX, (XXVIII), 31-35.

Fleurance, P., \& Pérez, S. (2008). Interrogations sur le métier d'entraîneur(e). Paris : INSEP.

Gould, D. (2016). Conducting Impactful Coaching Science Research: The Forgotten Role of Knowledge Integration and Dissemination. International Sport Coaching Journal, 3(2), 197-203.

Haugen, T. (2019). Key Success Factors for Merging Sport Science and Best Practice. International Journal of Sports Physiology and Performance, 1. https://doi.org/10.1123/ijspp.2019-0940

Jones, B., Till, K., Emmonds, S., Hendricks, S., Mackreth, P., Darrall-Jones, J., ... Rock, A. (2019). Accessing off-field brains in sport; an applied research model to develop practice. British Journal of Sports Medicine, 53(13), 791-793. https://doi.org/10.1136/bjsports-2016-097082

Lacourse, F., \& Thibault, R. (1996). Réflexion sur les conditions pour établir des liens entre praticiens et chercheurs. Nouveaux c@hiers de la recherche en éducation, 3(2), 295-310.

Lièvre, P. (2007). La construction de savoirs pour l'action par intégration de connaissances pratiques «tacites » et de savoirs scientifiques «classiques ». In M.-J. Avenier \& C. Schmitt (Eds.), La construction de savoirs pour l'action (p. 171-193). Paris : L'Harmattan.

Lyet, P. (2011). Traduction, transaction sociale et tiers intermédiaire dans les processus de collaboration de chercheurs et de praticiens dans le cadre de recherches-actions. Pensée plurielle, $n^{\circ} 28(3), 49$.

Mezirow, J. (2001). Penser son expérience: Une voie vers l'autoformation (Chronique sociale). Lyon : Chronique sociale.

Mouchet, A. (2018). L'expérience subjective en recherche et en formation. Villeneuve d'Ascq : Presses universitaires du Septentrion.

Pérez, S. (2009). Cognition et formation en sport de performance: De nouveaux cadres de pensée pour comprendre l'activité et la formation des cadres du sport de haut niveau? Intellectica, (52), 119-137.

Reade, I., Rodgers, W., \& Hall, N. (2008). Knowledge transfer: How do high performance coaches access the knowledge of sport scientists? International Journal of Sports Science and Coaching, 3(3), 319-334.

Reade, I., Rodgers, W., \& Spriggs, K. (2008). New ideas for high performance coaches: A case study of knowledge 
Actes de la 11ème Biennale de l'ARIS : Former des citoyens physiquement éduqués. Axe 3 - Au-delà de la performance, $10.25518 /$ sepaps 20.461

transfer in sport science. International Journal of Sports Science and Coaching, 3(3), 335-354.

Rolland, C., \& Cizeron, M. (2011). Comprendre et intervenir: Les connaissances des entraîneurs experts en gymnastique. @ctivités, 8(2), 53-76.

Saury, J. (2008). La coopération dans les situations d'intervention, de performance et d'apprentissage en contexte sportif (Habilitation à Diriger des Recherches, Université de Nantes, Nantes).

Schmitt, C. (2007). La communication de savoirs pour l'action. In M.-J. Avenier \& C. Schmitt (Eds.), La construction de savoirs pour l'action (p. 195-213). Paris : L'Harmattan.

Trudel, P., Milestetd, M., \& Culver, D. M. (2019). What the Empirical Studies on Sport Coach Education Programs in Higher Education Have to Reveal: A Review. International Sport Coaching Journal, 1, 1-13.

Vinatier, I., \& Rinaudo, J.-L. (2015). Rencontres entre chercheurs et praticiens: Quels enjeux? Carrefours de l'éducation, 39(1), 9-18. https://doi.org/10.3917/cdle.039.0009

Werthner, P., \& Trudel, P. (2006). A new theoretical perspective for understanding how coaches learn to coach. Sport psychologist, 20(2), 198-212.

PDF automatiquement généré le 2023-04-26 04:29:03

Url de l'article : https://popups.uliege.be/sepaps20/index.php?id=461

Publié par ULiège Library en Open Access et distribué suivant les termes et les conditions de la licence CC-BY (https://creativecommons.org/licenses/by/4.0/deed.fr) 